\title{
Smith-Purcell radiation from a charge moving above a finite-length grating
}

\author{
Amit S. Kesar* \\ Plasma Science and Fusion Center, Massachusetts Institute of Technology, 167 Albany Street, Cambridge, Massachusetts 02139, USA
}

(Received 12 May 2005; published 8 July 2005)

\begin{abstract}
Smith-Purcell radiation (SPR), generated by an electron beam traveling above a grating, is characterized by a broad range of frequencies. The radiated wavelength depends on the angle of observation according to the SPR resonance relationship and the bandwidth is inversely proportional to the number of the grating grooves. A rigorous theoretical model of SPR from a three-dimensional bunch of relativistic electrons passing above a grating of finite length is presented by an electric-field integral equation method. The finite-length grating results are compared with the case of an infinitely long grating assumption in which periodic boundary conditions are rigorously applied and with a model based on the image-charge approximation. The SPR resonance relationship is the same in all three formalisms. Significant errors in the strength of the radiated energy are introduced by the two approximations. In particular, for gratings with less than $\sim 20$ periods, the image-charge approximation and the infinitely long grating assumption result in an order of magnitude too high and too low radiated energy per groove, respectively, in the plane transverse to the grating groove lines. Numerical examples are calculated for an $\sim 18 \mathrm{MeV}$ bunch traveling above different finite-length gratings with a period of $2.5 \mathrm{~mm}$.
\end{abstract}

DOI: $10.1103 /$ PhysRevSTAB.8.072801

\section{INTRODUCTION}

Smith-Purcell radiation (SPR) [1] is typically formed by a charge passing above a periodic grating. It is characterized by a broad spectrum of frequencies and can be used as a coherent frequency-locked terahertz source [2]. The SPR resonance relationship correlates the $n$th harmonic of the radiated wavelength $\lambda$ to the spatial observation angles $\theta$ and $\phi$ by

$$
\lambda=\frac{D_{g}}{n}\left(\beta^{-1}-\sin \theta \sin \phi\right),
$$

where $D_{g}$ is the grating period and $\beta=v_{x} / c=$ $\left(1-\gamma^{-2}\right)^{1 / 2}$ is the relativistic bunch velocity as shown in Fig. 1. Choosing the $y$ axis parallel with the grating groove lines, the components of the wave number $k=\omega / c$ are $k_{x}=k \sin \phi \sin \theta, k_{y}=k \cos \phi$, and $k_{z}=k \sin \phi \cos \theta$, where $\omega$ is the angular frequency.

The SPR is caused by diffraction of the charge freespace evanescent waves (wake fields) from the grating [3]. Under the condition of an infinitely long, periodic grating an exact model was derived by van den Berg. The radiated energy is calculated by solving two separated integral equations, each having a periodic Green's function, excited by the charge wake fields [4]. Based on van den Berg's method, Haeberlé et al. calculated the SPR for electrons with energies of $1-100 \mathrm{MeV}$ by solving either the integral equation or by using a variational or a modal expansion method in order to alleviate the calculation time [5].

Based on the image-charge approximation, an induced surface current model was developed by Walsh et al. for a strip grating [6], generalized by Brownell et al. for an

*Electronic address: a_kesar@mit.edu
PACS numbers: 41.60. $-\mathrm{m}$, 02.70.Dh, 42.25.Fx, 42.79.Dj

arbitrary shaped grating [7], and optimized for very high charge energies by Trotz et al. [8]. This model has the advantage of a short calculation time which is $\sim 10^{4}$ times shorter compared to the integral equation method.

Time- and frequency-domain models of SPR by a twodimensional bunch moving above a finite-length grating were derived in [9]. A very good agreement was obtained between these models. It was shown that the finite length of the grating has to be taken into account in most experiments and that in the limit of an infinitely long grating the results are consistent with van den Berg's line of charge model [10].

Exact calculation of the radiated energy is important for terahertz generation as well as for bunch-length diagnostics. In many of the SPR experiments, the radiation was compared to theory based on either van den Berg's model [11-13] or the image-charge model [14-18]. A good agreement with the image-charge model was reported in

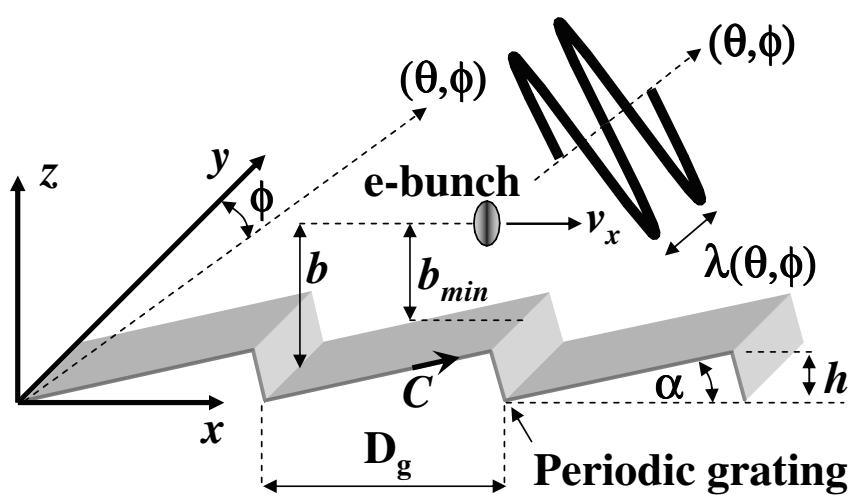

FIG. 1. The SPR scheme. An electron bunch is traveling at an axial velocity $v_{x}$ above an echelle grating of period $D_{g}$. 
[16,18] for beam energies of 1.5-2.3 MeV. However, several orders of magnitude difference for the calculated grating efficiency by the two approaches at a beam energy of $855 \mathrm{MeV}$ was found in [13].

SPR can be used as a nondestructive diagnostic method to measure short bunch lengths $[19,20]$. Subpicosecond bunch-length measurements were obtained by measuring the SPR from $15 \mathrm{MeV}$ bunches [2,21]. A very good agreement was obtained, on a relative scale (peak energy normalized to unity), between the measured radiation patterns and the calculations using the three-dimensional electricfield integral equation (EFIE) model described in this paper. This comparison allowed to evaluate bunch lengths of $1.0 \pm 0.1 \mathrm{ps}$ and $0.6 \pm 0.1 \mathrm{ps}$ at different accelerator operating parameters. The measured bunch lengths agreed very well with independent measurements by a circularly polarized deflector $[22,23]$.

The objectives of this paper are to (a) extend the twodimensional EFIE model in [9] to the general case of a three-dimensional bunch moving above a finite-length grating, (b) demonstrate consistency with van den Berg's model [4] for the special case of an infinitely long periodic grating assumption, and (c) compare the radiated energy per groove calculated by the finite-length grating, van den Berg, and the image-charge models. A preliminary work on the three-dimensional EFIE model described in this paper is reported in [24].

\section{EFIE FORMULATION}

\section{A. Finite-length grating}

In this subsection electric-field integral equations are derived for Smith-Purcell radiation by a three-dimensional bunch moving above a finite-length grating. Assuming a metal grating as a reflector, the reflected-field vector potential $\mathbf{A}(\mathbf{r}, \omega)$ at each angular frequency $\omega$ is correlated to the surface current $\mathbf{J}(\mathbf{r}, \omega)$ on the perimeter of the reflector by [25]

$$
\mathbf{A}(\mathbf{r}, \omega)=\int_{C} \int_{-W / 2}^{W / 2} \mathbf{J}\left(\mathbf{r}^{\prime}, \omega\right) G^{3 D}\left(\mathbf{r}-\mathbf{r}^{\prime}, \omega\right) d y^{\prime} d c^{\prime},
$$

where the grating groove lines are parallel to the $y$ direction and are of width $W$. The integration path along the grating profile at the $x z$ plane is denoted by $C$, as described in Fig. 1. In this equation the three-dimension Green's function is

$$
G^{3 D}\left(\mathbf{r}-\mathbf{r}^{\prime}, \omega\right)=\frac{e^{-j k\left|\mathbf{r}-\mathbf{r}^{\prime}\right|}}{4 \pi\left|\mathbf{r}-\mathbf{r}^{\prime}\right|},
$$

where a frequency dependence $e^{j \omega t}$ is assumed and the observation and source points are $\mathbf{r}=x \hat{x}+y \hat{y}+z \hat{z}$ and $\mathbf{r}^{\prime}=x^{\prime} \hat{x}+y^{\prime} \hat{y}+z^{\prime} \hat{z}$, respectively.

The electric component of the reflected field is given by

$$
\mathbf{E}^{\mathrm{ref}}(\mathbf{r}, \omega)=-j \frac{Z_{0}}{k}\left(\nabla \nabla+k^{2}\right) \mathbf{A}(\mathbf{r}, \omega),
$$

where $Z_{0}=\left(\mu_{0} / \epsilon_{0}\right)^{1 / 2}$ is the free-space impedance and $\mu_{0}$ and $\epsilon_{0}$ are the free-space permeability and permittivity, respectively. Assuming a perfectly conducting grating, the tangential component of the electric field $\mathbf{E}_{\|}=$ $\mathbf{E}^{\text {inc }}+\mathbf{E}^{\text {ref }}=0$ on the grating surface. The incident electric field is given by the Fourier transform of the free-space electric field produced by a charge $q$ moving along the $x$ direction at a relativistic velocity $\beta$

$$
\begin{aligned}
\mathbf{E}^{\text {inc }}(\mathbf{r}, \omega)= & \frac{q Z_{0}}{2 \pi \beta \gamma} e^{-j(k / \beta)\left(x-x_{0}\right)} \frac{k}{\beta} \\
& \times\left[\frac{\hat{y}\left(y-y_{0}\right)+\hat{z}\left(z-z_{0}\right)}{\rho} K_{1}\left(\frac{k \rho}{\beta \gamma}\right)\right. \\
& \left.+j \frac{\hat{x}}{\gamma} K_{0}\left(\frac{k \rho}{\beta \gamma}\right)\right],
\end{aligned}
$$

where $\left(x_{0}, y_{0}, z_{0}\right)$ are the charge coordinates, $\rho=$ $\left[\left(y-y_{0}\right)^{2}+\left(z-z_{0}\right)^{2}\right]^{1 / 2}$ is the transverse distance, and $K_{0}$ and $K_{1}$ are the zeroth and first-order modified Bessel functions of the second kind, respectively.

Equations (2)-(5) can be combined into a twodimensional electric-field integral equation solved along the grating profile and width to find the unknown surface current,

$$
\begin{aligned}
\mathbf{E}^{\mathrm{inc}}(\mathbf{r}, \omega)= & j \frac{Z_{0}}{k}\left(\nabla \nabla+k^{2}\right) \int_{C} \int_{-W / 2}^{W / 2} \mathbf{J}\left(\mathbf{r}^{\prime}, \omega\right) \\
& \times G^{3 D}\left(\mathbf{r}-\mathbf{r}^{\prime}, \omega\right) d y^{\prime} d c^{\prime},
\end{aligned}
$$

where the source and observation points are located on the grating surface. For $k \rho / \beta \gamma>1$ the modified Bessel functions in Eq. (5) are dominated by an exponential decay. The induced surface current is expected to decay similarly, and thus, for a grating of sufficient width $W / 2 \gg \beta \gamma / k$ and $W / 2 \gg b$, where $b=b_{\min }+h$ and $b_{\min }$ is the average height of the bunch above the grating as shown in Fig. 1, Eq. (6) could be approximated by

$$
\begin{aligned}
\mathbf{E}^{\text {inc }}(\mathbf{r}, \omega) \approx & j \frac{Z_{0}}{k}\left(\nabla \nabla+k^{2}\right) \int_{C} \int_{-\infty}^{\infty} \mathbf{J}\left(\mathbf{r}^{\prime}, \omega\right) \\
& \times G^{3 D}\left(\mathbf{r}-\mathbf{r}^{\prime}, \omega\right) d y^{\prime} d c^{\prime} .
\end{aligned}
$$

Applying the spatial Fourier operator $\int_{-\infty}^{\infty} e^{j k_{y} y} d y$ on Eq. (7) results for $k_{y}<k$ in two coupled one-dimension electric-field integral equations, solved along the grating profile

$$
\begin{aligned}
& \tilde{E}_{c}^{\text {inc }}\left(x, z, k_{y}, \omega\right)=j \frac{Z_{0}}{k} \int_{C}\left[\tilde{J}_{c} \mathcal{A} \tilde{G}+\tilde{J}_{y} \mathcal{B} \tilde{G}\right] d c^{\prime} \\
& \tilde{E}_{y}^{\text {inc }}\left(x, z, k_{y}, \omega\right)=j \frac{Z_{0}}{k} \int_{C}\left[\tilde{J}_{c} C \tilde{G}+\tilde{J}_{y} \mathcal{D} \tilde{G}\right] d c^{\prime} .
\end{aligned}
$$

For a charged bunch having a transverse and longitudinal distribution function $f\left(x_{0}, y_{0}, z_{0}\right)$ traveling above the grating, i.e. $z_{0}>h$ for all of the particles in the bunch where $h$ is the grating height as shown in Fig. 1, the spatial electricfield incident on the grating is 


$$
\begin{aligned}
\tilde{\mathbf{E}}^{\text {inc }}\left(x, z, k_{y}, \omega\right)= & \frac{q Z_{0}}{2 \beta} e^{-j(k / \beta) x+k_{a} z}\left[\hat{y} \frac{j k_{y}}{k_{a}}-\hat{z}\right. \\
& \left.+j \hat{x} \frac{k / \beta \gamma}{\gamma k_{a}}\right] F(k),
\end{aligned}
$$

where $k_{a}=\sqrt{(k / \beta \gamma)^{2}+k_{y}^{2}}$ and the bunch form factor, $F(k)=\int \mathbb{\int} e^{j(k / \beta) x_{0}+j k_{y} y_{0}-k_{a} z_{0}} f\left(x_{0}, y_{0}, z_{0}\right) d x_{0} d y_{0} d z_{0}$, affects the coupling of the wake to the grating and produces the cutoff frequency. Throughout this paper the bunch longitudinal distribution is assumed to be Gaussian with a full width at half maximum (FWHM) of $\sigma_{x}$ and the transverse distribution is assumed as a $\delta$ function at $y_{0}=0$ and $z_{0}=$ $b$, namely, $F(k)=\exp \left(-k_{a} b-(k / \beta)^{2} \sigma_{x}^{2} / 16 \ln 2\right)$.

The spatial electric-field components tangent to the grating profile and along the grating grooves are $\tilde{E}_{c}^{\text {inc }}\left(x, z, k_{y}, \omega\right)=\tilde{E}_{x}^{\text {inc }}\left(x, z, k_{y}, \omega\right) \cos (\alpha)+\tilde{E}_{z}^{\text {inc }}\left(x, z, k_{y}, \omega\right) \times$ $\sin (\alpha)$ and $\tilde{E}_{y}^{\text {inc }}\left(x, z, k_{y}, \omega\right)$, respectively, the corresponding spatial components of the surface current are $\tilde{J}_{c}\left(x^{\prime}, z^{\prime}, k_{y}, \omega\right)=\tilde{J}_{x}\left(x^{\prime}, z^{\prime}, k_{y}, \omega\right) \cos \left(\alpha^{\prime}\right)+\tilde{J}_{z}\left(x^{\prime}, z^{\prime}, k_{y}, \omega\right) \times$ $\sin \left(\alpha^{\prime}\right)$ and $\tilde{J}_{y}\left(x^{\prime}, z^{\prime}, k_{y}, \omega\right)$, respectively, where $\alpha$ and $\alpha^{\prime}$ are the observation and source angles tangent to the grating profile, respectively. The twodimensional free-space Green's function is $\tilde{G}=$ $(1 / 4 j) H_{0}^{(2)}\left[k_{\perp} \sqrt{\left(x-x^{\prime}\right)^{2}+\left(z-z^{\prime}\right)^{2}}\right] \quad$ where $\quad k_{\perp}=$ $\sqrt{k^{2}-k_{y}^{2}}$ and the operators acting on it are

$$
\begin{aligned}
\mathcal{A}= & k^{2} \cos \left(\alpha-\alpha^{\prime}\right)+\cos \alpha \cos \alpha^{\prime} \partial_{x}^{2}+\sin \alpha \sin \alpha^{\prime} \partial_{z}^{2} \\
& +\sin \left(\alpha+\alpha^{\prime}\right) \partial_{x z} \\
\mathcal{B}= & -j k_{y}\left(\cos \alpha \partial_{x}+\sin \alpha \partial_{z}\right) \\
C & -j k_{y}\left(\cos \alpha^{\prime} \partial_{x}+\sin \alpha^{\prime} \partial_{z}\right) \\
\mathcal{D}= & k_{\perp}^{2}
\end{aligned}
$$

The special case of $k_{y}=0$ results in $\tilde{E}_{y}^{\text {inc }}=0$ and Eqs. (8a) and (8b) are reduced to $\tilde{J}_{c}$ as a function of $\tilde{E}_{c}^{\text {inc }}$, in agreement with the two-dimensional $\mathrm{TE}_{y}$ polarized SPR described in [9].

The unknown surface currents in Eqs. (8a) and (8b) were solved by dividing the grating profile into $N$ straight segments of $\Delta_{n}$ length and assuming a piecewise constant current in each one,

$$
\begin{aligned}
& \tilde{J}_{c}\left(x, z, k_{y}, \omega\right) \approx \sum_{n=1}^{N} a_{n}\left(k_{y}, \omega\right) g_{n}(x, z) \\
& \tilde{J}_{y}\left(x, z, k_{y}, \omega\right) \approx \sum_{n=1}^{N} b_{n}\left(k_{y}, \omega\right) g_{n}(x, z),
\end{aligned}
$$

where $g_{n}(x, z)=1$ at the $n$th segment and zero out of it. Thus, Eqs. (8a) and (8b) were approximated by a set of $2 \mathrm{~N}$ linear equations

$$
\left[\begin{array}{l}
E_{c m} \\
E_{y m}
\end{array}\right]=\left[\begin{array}{ll}
A_{m n} & B_{m n} \\
C_{m n} & D_{m n}
\end{array}\right]\left[\begin{array}{l}
a_{n} \\
b_{n}
\end{array}\right]
$$

where $E_{c m}$ and $E_{y m}$ are the incident electric components given by Eq. (9) at the center of the $m$ th observation segment. The matrix diagonal terms, related to the Hankel function singularity, are given by the closed form [25]

$$
\begin{aligned}
A_{n n}= & \frac{Z_{0} k_{\perp}^{2} \Delta_{n}}{8 k}\left\{1-\frac{j}{\pi}\left[2 \ln \left(\frac{1.781 k_{\perp} \Delta_{n}}{4}\right)-3\right.\right. \\
& \left.\left.+\frac{16}{\left(k_{\perp} \Delta_{n}\right)^{2}}\right]\right\}+\left(k_{y} / k_{\perp}\right)^{2} D_{n n}, \\
B_{n n}= & 0, \\
C_{n n}= & 0, \\
D_{n n}= & \frac{Z_{0} k_{\perp}^{2} \Delta_{n}}{4 k}\left\{1-\frac{j}{2 \pi}\left[2 \ln \left(\frac{1.781 k_{\perp} \Delta_{n}}{4}\right)-1\right]\right\},
\end{aligned}
$$

and the other terms are calculated numerically.

The far-field vector potential approximated for a large argument $\mathbf{r} \gg \mathbf{r}^{\prime}$ is

$$
\begin{aligned}
\mathbf{A}^{\operatorname{far}}(r, \theta, \phi, \omega) \simeq & \frac{e^{-j k r}}{4 \pi r} \int_{C}\left[\int_{-W / 2}^{W / 2} \mathbf{J}\left(\mathbf{r}^{\prime}, \omega\right) e^{j k_{y} y^{\prime}} d y^{\prime}\right] \\
& \times e^{j k_{x} x^{\prime}+j k_{z} z^{\prime}} d c^{\prime},
\end{aligned}
$$

were $\quad k_{x}=k \sin \phi \sin \theta, \quad k_{y}=k \cos \phi, \quad$ and $\quad k_{z}=$ $k \sin \phi \cos \theta$. Under the assumption of a sufficient grating width $\quad \int_{-W / 2}^{W / 2} \mathbf{J}\left(\mathbf{r}^{\prime}, \omega\right) e^{j k_{y} y^{\prime}} d y^{\prime} \approx \int_{-\infty}^{\infty} \mathbf{J}\left(\mathbf{r}^{\prime}, \omega\right) e^{j k_{y} y^{\prime}} d y^{\prime}=$ $\tilde{\mathbf{J}}\left(x^{\prime}, z^{\prime}, k_{y}, \omega\right)$, Eq. (14) becomes

$$
\mathbf{A}^{\mathrm{far}}(r, \theta, \phi, \omega) \simeq \frac{e^{-j k r}}{4 \pi r} \int_{C} \tilde{\mathbf{J}}\left(x^{\prime}, z^{\prime}, k_{y}, \omega\right) e^{j k_{x} x^{\prime}+j k_{z} z^{\prime}} d c^{\prime},
$$

where $\tilde{\mathbf{J}}\left(x^{\prime}, z^{\prime}, k_{y}, \omega\right)=\hat{x} \tilde{J}_{c} \cos \alpha^{\prime}+\hat{y} \tilde{J}_{y}+\hat{z} \tilde{J}_{c} \sin \alpha^{\prime}$. The magnetic component of the far field is approximated by

$$
\mathbf{H}(r, \theta, \phi, \omega) \simeq-j \mathbf{k} \times \mathbf{A}^{\mathrm{far}},
$$

and the power spectrum is

$$
P_{s}(\theta, \phi, \omega)=Z_{0} r^{2}|\mathbf{H}(r, \theta, \phi, \omega)|^{2} .
$$

The angular distribution of the average radiated energy per groove is given by Parseval's theorem,

$$
E_{A V}(\theta, \phi)=\frac{1}{N_{g} \pi} \int_{0}^{\infty} P_{s}(\theta, \phi, \omega) d \omega,
$$

where for coherent radiation it is sufficient to calculate up to a maximum frequency of $\omega=2 \pi c / \sigma_{x}$ where the bunch form factor is very small. The integration range in Eq. (18) can be varied in order to calculate the energy in a specific range of frequencies for each observation angle, in order to obtain the contribution from a given SPR order, such as $0.5 \omega_{n}<\omega<1.5 \omega_{n}$, where $\omega_{n}=2 \pi n c / D_{g}\left(\beta^{-1}-\right.$ $\sin \theta \sin \phi$ ).

\section{B. Periodic solution}

In this subsection the finite-length formulation is rederived for the special case of an infinitely long periodic 
structure in order to validate its consistency with van den Berg's model [4]. In an infinitely long periodic grating $\tilde{\mathbf{J}}\left(x+p D_{g}, z, k_{y}, \omega\right)=\tilde{\mathbf{J}}\left(x, z, k_{y}, \omega\right) e^{-j k_{x 0} p D_{g}}$, where $k_{x 0}=k / \beta$ is the bunch free-space wave number determined by its velocity in the $\hat{x}$ direction.

The periodic EFIE has a form similar to Eqs. (8a) and (8b) with the difference of being solved along a single grating period $D_{g}$,

$$
\begin{aligned}
& \tilde{E}_{c}^{\mathrm{inc}}\left(x, z, k_{y}, \omega\right)=j \frac{Z_{0}}{k} \int_{D_{g}}\left[\tilde{J}_{c} \mathcal{A} \tilde{G}_{p}+\tilde{J}_{y} \mathcal{B} \tilde{G}_{p}\right] d c^{\prime} \\
& \tilde{E}_{y}^{\mathrm{inc}}\left(x, z, k_{y}, \omega\right)=j \frac{Z_{0}}{k} \int_{D_{g}}\left[\tilde{J}_{c} C \tilde{G}_{p}+\tilde{J}_{y} \mathcal{D} \tilde{G}_{p}\right] d c^{\prime},
\end{aligned}
$$

and a periodic Green's function $\tilde{G}_{p}=(1 / 4 j) \times$ $\sum_{p=-\infty}^{\infty} H_{0}^{(2)}\left(k_{\perp} \sqrt{\left(x-x^{\prime}-p D_{g}\right)^{2}+\left(z-z^{\prime}\right)^{2}}\right) e^{-j k_{x 0} p D_{g}} \quad$ replacing the free-space Green's function in Eqs. (8a) and (8b). The periodic surface currents in Eqs. (19a) and (19b) are found similarly, as described in the previous subsection, by dividing the single-period integration into linear segments and approximating the currents as sums of piecewise constant functions. Because of the slow convergence of the periodic Hankel function, the following procedure was used to accelerate its evaluation [26,27]:

$$
\begin{gathered}
\frac{1}{4 j} \sum_{p=-\infty}^{\infty} H_{0}^{(2)}\left(k_{\perp} \sqrt{\left(x-x^{\prime}-p D_{g}\right)^{2}+\left(z-z^{\prime}\right)^{2}}\right) e^{-j k_{x 0} p D_{g}}=\frac{1}{4 j} H_{0}^{(2)}\left(k_{\perp} \sqrt{\left(x-x^{\prime}\right)^{2}+\left(z-z^{\prime}\right)^{2}}\right) \\
+\frac{e^{-j k_{\perp}\left(x-x^{\prime}\right)}}{\pi} \int_{0}^{\infty} \frac{\exp \left[-k_{\perp}\left(D_{g}+x-x^{\prime}\right) u^{2}-j\left(k_{\perp}-k_{x 0}\right) D_{g}\right] \cos \left[k_{\perp}\left(z-z^{\prime}\right) u \sqrt{u^{2}+2 j}\right]}{\left\{1-\exp \left[-k_{\perp} D_{g} u^{2}-j\left(k_{\perp}-k_{x 0}\right) D_{g}\right]\right\} \sqrt{u^{2}+2 j}} d u \\
+\frac{e^{j k_{\perp}\left(x-x^{\prime}\right)}}{\pi} \int_{0}^{\infty} \frac{\exp \left[-k_{\perp}\left(D_{g}-x+x^{\prime}\right) u^{2}-j\left(k_{\perp}+k_{x 0}\right) D_{g}\right] \cos \left[k_{\perp}\left(z-z^{\prime}\right) u \sqrt{u^{2}+2 j}\right]}{\left\{1-\exp \left[-k_{\perp} D_{g} u^{2}-j\left(k_{\perp}+k_{x 0}\right) D_{g}\right]\right\} \sqrt{u^{2}+2 j}} d u,
\end{gathered}
$$

where the right-hand-side first term is related to $p=0$ and the other two are related to the summation of $p=$ $-\infty, \ldots,-1$, and $p=1, \ldots, \infty$, respectively. The advantage of this procedure is that the self-term argument singularities are evaluated as in Eqs. (13a)-(13d), and the partial derivatives in Eqs. (10a)-(10d) can be applied analytically on the second and third terms of Eq. (20).

Defining axial and transverse periodic wave numbers $k_{x n}=k_{x}+2 \pi n / D_{g}$ and $k_{z n}=\left(\sqrt{k_{\perp}^{2}-k_{x n}^{2}}\right)^{*}$, respectively, where ( $)^{*}$ denotes a complex conjugate value, the periodic vector potential can be represented as a sum over the propagating Floquet harmonics $\operatorname{Re}\left(k_{z n}\right) \geq 0$ which correspond to the spectral grating orders:

$$
\begin{aligned}
\mathbf{A}\left(x, z, k_{y}, \omega\right) & =\sum_{\operatorname{Re}\left(k_{z n}\right)>0} \mathbf{A}_{n}\left(x, z, k_{y}, \omega\right) \\
& =\int_{D_{g}} \tilde{\mathbf{J}}\left(x^{\prime}, z^{\prime}, k_{y}, \omega\right) \sum_{\operatorname{Re}\left(k_{z n}\right)>0} \tilde{G}_{p n} d c^{\prime}
\end{aligned}
$$

In this equation the periodic Green's function is represented by its Poisson sum transformation [26]

$$
\tilde{G}_{p}=\sum_{n=-\infty}^{\infty} \tilde{G}_{p n}=\sum_{n=-\infty}^{\infty}\left[\frac{e^{-j k_{x n}\left(x-x^{\prime}\right)-j k_{z n}\left|z-z^{\prime}\right|}}{2 j D_{g} k_{z n}}\right] .
$$

Thus, for observation points above the grating $(z>b)$ and real values of $k_{z n}$, the $n$th SPR order of the magnetic field is

$$
\begin{aligned}
\mathbf{H}_{n}\left(x, z, k_{y}, \omega\right)= & -\frac{e^{-j k_{x n} x-j k_{z n} z}}{2 D_{g} k_{z n}} \int_{D_{g}} \mathbf{k}_{n} \\
& \times \tilde{\mathbf{J}}\left(x^{\prime}, z^{\prime}, k_{y}, \omega\right) e^{j k_{x n} x^{\prime}+j k_{z n} z^{\prime}} d c^{\prime},
\end{aligned}
$$

where $\mathbf{k}_{n}=\hat{x} k_{x n}+\hat{y} k_{y}+\hat{z} k_{z n}$. Wood-Rayleigh anomalies [28] are expected at wavelengths satisfying $k_{z n}^{2}=k^{2}-$ $k_{y}^{2}-k_{x n}^{2}=0$, where the wave numbers are functions of $\theta$ and $\phi$ according to the SPR relationship described in Eq. (1). These anomalies will appear at real angles fulfilling

$$
\theta_{n}=\sin ^{-1}\left[\frac{\beta^{-1}(n-1)-\sin \phi}{n \sin \phi}\right]
$$

where $0^{\circ}<\phi<180^{\circ}$ and $n=2,3, \ldots, \infty$.

The $n$ th-order radiated energy per groove is found from Eq. (23) as

$$
E_{n}(\theta, \phi)=\frac{2 n^{2}}{\epsilon_{0} D_{g}}\left|\mathbf{H}_{n}\left(k_{y}, \omega\right)\right|^{2} \frac{\cos ^{2} \theta \sin ^{2} \phi}{\left(\beta^{-1}-\sin \theta \sin \phi\right)^{3}},
$$

where $\left|\mathbf{H}_{n}\left(k_{y}, \omega\right)\right|^{2}=\left|H_{x n}\right|^{2}+\left|H_{y n}\right|^{2}+\left|H_{z n}\right|^{2}$ is independent of the $(x, z)$ coordinates, $k_{y}=k \cos \phi$, and $\omega$ is related to $\theta$ and $\phi$ by Eq. (1).

\section{NUMERICAL EXAMPLE}

A numerical example of SPR from a finite-length echelle grating is presented in the following section. The bunch and grating parameters are as listed in Table I, unless specified otherwise. 
TABLE I. Smith-Purcell radiation parameters.

Bunch charge $q$

Bunch relativistic factor $\gamma$

Height above the grating, $b_{\min }$

Bunch length $\sigma_{x}$

Grating period $D_{g}$

Blaze angle $\alpha$

Number of periods, $N_{g}$

EFIE resolution $\Delta_{n}$

The surface currents along the 20-period grating profile, $\tilde{J}_{c}(x, z)$ and $\tilde{J}_{y}(x, z)$, calculated at a Fourier frequency of $120 \mathrm{GHz}$ and at $\phi=85^{\circ}$, are presented in Fig. 2 by solid and dashed lines, respectively. These currents are normalized to a unit charge of $q=1 \mathrm{C}$ and a bunch form factor of $F(k)=1$. The inset shows the corresponding currents along one period of an infinitely long grating. It is seen that the 20-period currents, $\tilde{J}_{c}$ and $\tilde{J}_{y}$, have a general structure per groove which is similar to the infinitely long currents, respectively. However, the envelopes of the 20 -period $\tilde{J}_{c}$ and $\tilde{J}_{y}$ are large in the first groove. These envelopes approach the values of the infinitely long currents, respectively, toward the last groove.

The solution of the average first-order $(n=1)$ radiated energy per groove $E_{A V}(\theta, \phi)$ is shown in Fig. 3 as contours in $\mathrm{nJ} / \mathrm{sr}$ vs $\theta$ and $\phi$. The calculation is symmetric around $\phi=90^{\circ}\left(k_{y}=0\right)$, thus $E_{A V}(\theta, 180-\phi)=E_{A V}(\theta, \phi)$.

The average first-order radiated energy per groove by the 20-period grating is shown in Figs. 4(a) (solid line) and 4(b) for angular ranges of $-60^{\circ} \leq \theta \leq 60^{\circ}, \phi=90^{\circ}$ and $\theta=0^{\circ}, 30^{\circ} \leq \phi \leq 90^{\circ}$, respectively. This energy is compared to the first-order radiated energy per groove calcu-

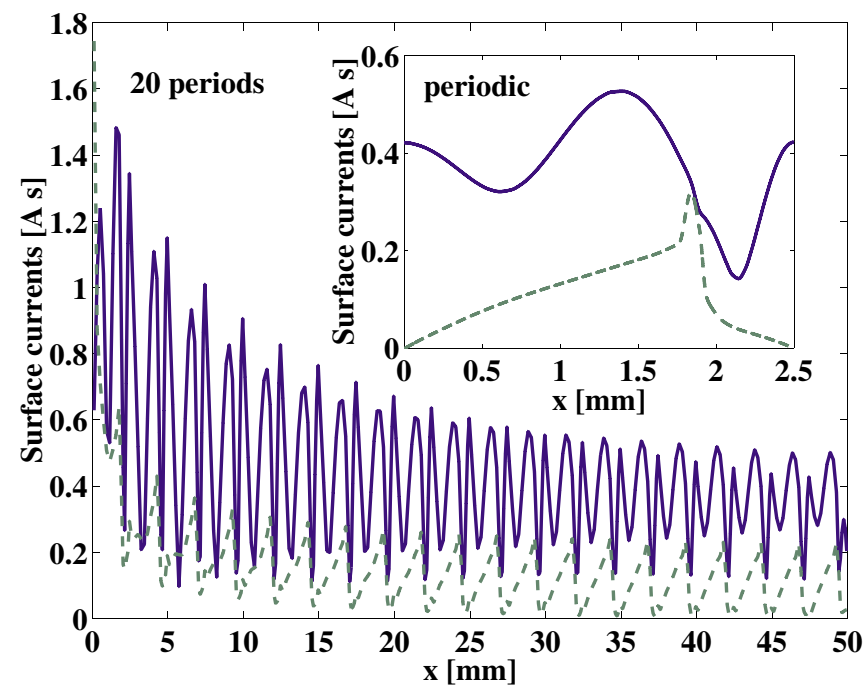

FIG. 2. (Color) Surface currents $\tilde{J}_{c}$ (thin line) and $\tilde{J}_{y}$ (thick line) along the profile of the 20-period grating at a Fourier frequency of $120 \mathrm{GHz}$ and at $\phi=85^{\circ}$. Inset: corresponding surface currents by the periodic solution plotted along a single period.

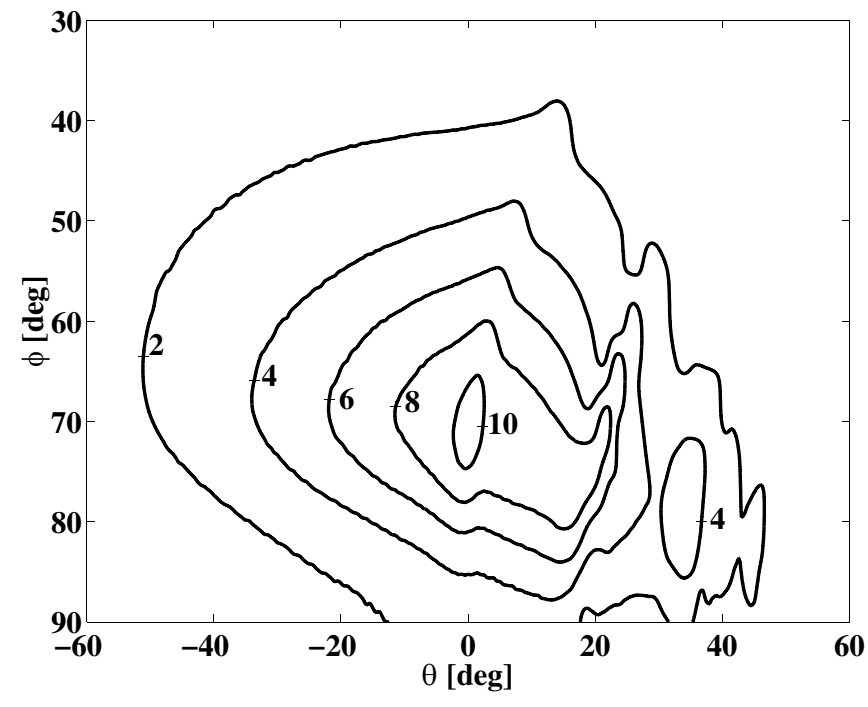

FIG. 3. Contour plot of the first-order average radiated energy per groove in $\mathrm{nJ} / \mathrm{sr}$ vs observation angles $\theta$ and $\phi$.

lated by van den Berg's model for an infinitely long grating [4] (dotted line) and by the surface current model which is based on the image-charge approximation [7] (dashed line). Under the infinitely long grating assumption, the periodic EFIE results (not shown) agree with van den Berg's model with $<10 \%$ difference between the two, thus demonstrating that the EFIE model is consistent with van den Berg's model.

The sharp energy variations seen in the periodic solution in Fig. 4(a) are caused by the Wood-Rayleigh anomalies. Following [5], the radiated energy was calculated at steps of $1^{\circ}$, except near these anomalies, where offsets of $\pm\left(10^{-7}, 10^{-4}, 10^{-3}\right)$ from the angles calculated by Eq. (24) were used. In Fig. 3, it is seen that the energy contours curve strongly in the region $20^{\circ}<\theta<30^{\circ}$ and $50^{\circ}<\phi<70^{\circ}$. The locations of these energy variations agree with the predicted values of the Wood-Rayleigh anomalies described in Eq. (24) except that for the finitelength grating they do not show up near $\phi=90^{\circ}$.

It is seen in Fig. 4(a) that the image-charge approximation and the infinite-length assumption result in an energy per groove which is an order of magnitude too high and too low, respectively, compared to the finite-length EFIE calculation. Figure 4(b) shows that for $\phi \lesssim 60^{\circ}$ (i.e. $k_{y} / k \gtrsim$ 0.5 ), the difference between the energy per groove emitted from the 20-period and the infinitely long grating becomes smaller.

In addition to the consistency between the periodic EFIE and van den Berg's model, the minimum number of grooves $N_{\min }$ to provide less than $10 \%$ difference between the radiated energy per groove by the finite-length and the infinitely long grating was calculated. It was found that $N_{\text {min }}$ was strongly dependent on $\phi$. For $\phi=90^{\circ}\left(k_{y}=0\right)$ it required more than 250 grooves, in agreement with the convergence described in [9]. However, for $\phi=80^{\circ}, 70^{\circ}$, and $60^{\circ}, N_{\min }$ was $\sim 100,20$, and 10 , respectively. 

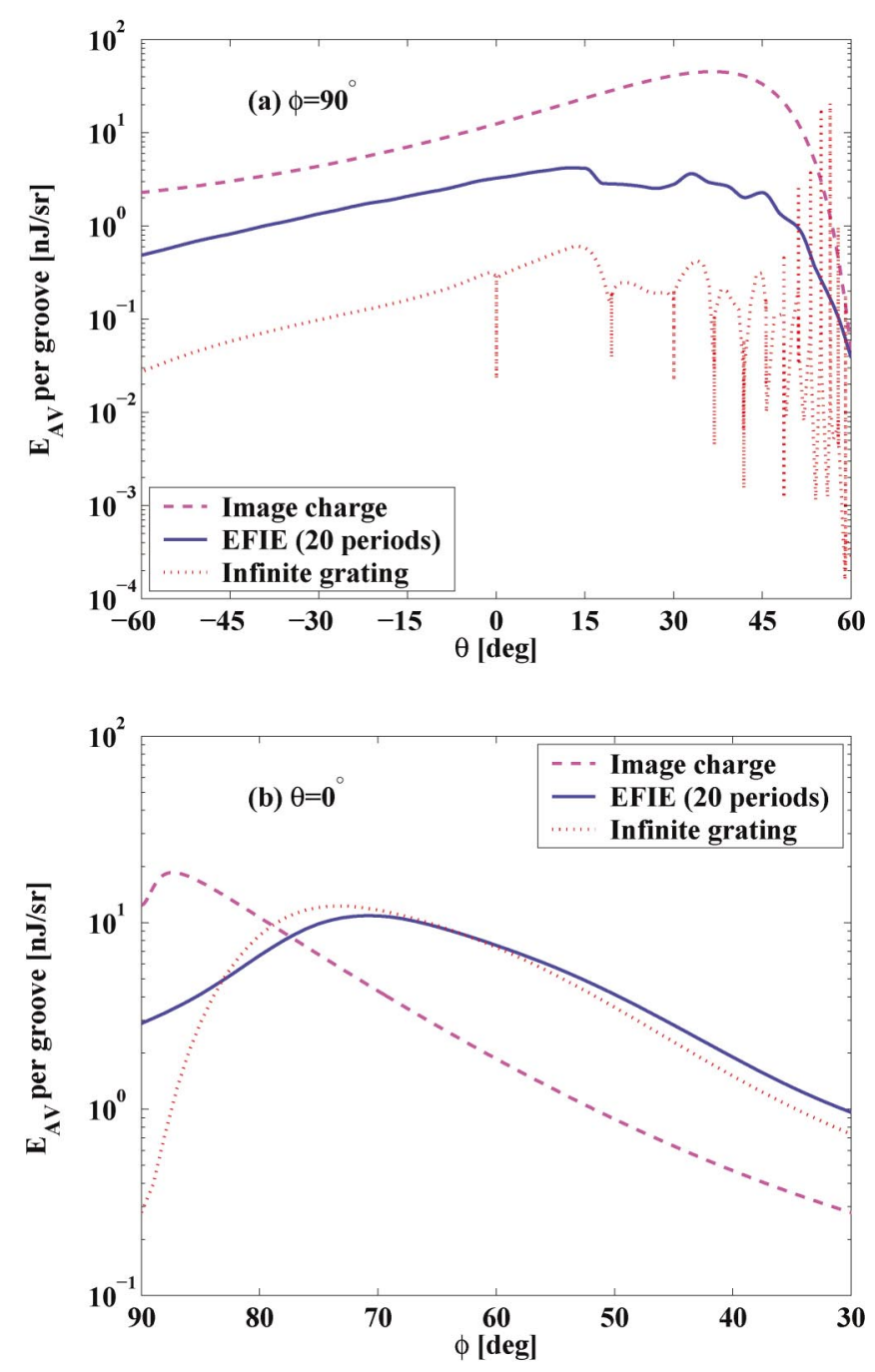

FIG. 4. (Color) Comparison of average first-order radiated energy per groove by the 20 -period grating (solid line) with the energy per groove by the image-charge model (dashed line) and by the infinitely long grating assumption (dotted line). The energies are plotted versus $\theta$ when $\phi=90^{\circ}$ (a) and versus $\phi$ when $\theta=0^{\circ}(\mathrm{b})$.

In order to illustrate bunch-length measurement by Smith-Purcell radiation, the first-order average radiated energy per groove vs observation angle $\theta$ (when $\phi=$ $90^{\circ}$ ) was calculated for bunch lengths of 300,360 , and $420 \mu \mathrm{m}$. The results are presented in Fig. 5 by solid, dashed, and dotted lines, respectively. As expected from the bunch form factor, $F(k)$, as the bunch gets longer the radiation at large observation angles decreases more than at small angles. Such a plot could be used to determine the bunch length by comparing the measured radiation pattern to several theoretical curves, as in [21].

\section{DISCUSSION}

The EFIE model in [9] was extended to the threedimensional case of a bunch moving above a finite-length

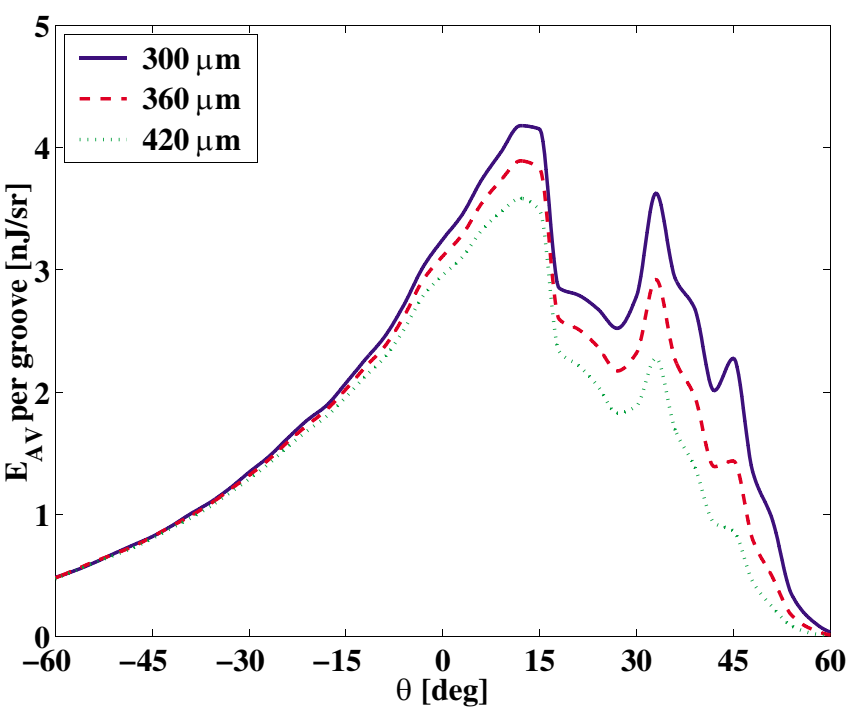

FIG. 5. (Color) First-order average radiated energy per groove vs observation angle $\theta$ (when $\phi=90^{\circ}$ ), plotted for bunch lengths of $300 \mu \mathrm{m}$ (solid line), $360 \mu \mathrm{m}$ (dashed line), and $420 \mu \mathrm{m}$ (dotted line).

grating. The results were compared to those of an infinitely long grating in which periodic boundary conditions are assumed [4] and to those by the image-charge approximation [7]. A considerable error in the strength of the radiated energy per groove is introduced by either assumption, and especially for transverse angles $\phi \sim 90^{\circ}$. The results by the EFIE model approach those by van den Berg [4] when $N_{g}$ is increased. In addition, using an infinitely long grating approximation the periodic EFIE is consistent with [4].

For $k_{y} \geq 0.5$ good agreement is obtained between the finite-length and the infinitely long approaches for gratings as short as ten grooves. This agreement could be intuitively understood by the coupling between the propagating wakefield and the reflected waves as the bunch moves above the grating [9]. As the bunch is passing above the first groove it induces a surface current. This current generates reflected fields. The radiation towards the $x$ direction induces current on the second groove which occurs, for relativistic velocities, at about the same time as the bunch is above the second groove, thus, changing the amount of current on the second groove. The radiation from the $n+1$ groove is therefore different from that of the previous one until the solution approaches a stable value, as seen in Fig. 2. Thus, a maximum coupling occurs for $k_{x 0}-k_{\perp} \rightarrow 0$. This explains the large error by the periodic solution at $\phi=90^{\circ}$ as well as the reason for not seeing the Wood-Rayleigh anomalies in Fig. 4(a).

The image-charge approximation resulted in an order of magnitude difference in the radiated energy, as shown in Figs. 4(a) and 4(b). Six orders of magnitude difference were reported by Kube et al. [13] comparing the SPR experimental results from an electron beam energy of $855 \mathrm{MeV}$ to a surface current model similar to the 
image-charge approximation by Brownell et al. [7], while fair agreement was obtained with van den Berg's model. A possible reason for the inaccuracy of the image-charge approximation for high beam energies may be due to the scale of the beam height above the grating compared to the length of each facet of the grating. The electrostatic limit of a stationary charge requires a perfectly conducting facet to be an order of magnitude longer than the height of the charge above it in order that most of the field lines from the charge will connect through the metal to form the image below it. As the charge travels at a relativistic velocity $\gamma$, the time required for the information to be updated to form the exact image is $\gamma$ times longer. Thus, a failure to include that may result in an error on the order of $\gamma^{2}$ higher energy, in agreement with [13].

The EFIE model in this paper assumes a grating of sufficient width $W / 2 \gg \beta \gamma / k$ and $W / 2 \gg b$. The radiation from a narrow grating which does not meet these criteria is expected to be lower in energy because only part of the bunch transverse evanescent wave would be diffracted from the grating. In addition, the radiation pattern is expected to become broader over the transverse observation angle $\phi$.

While a bunch-length measurement was obtained in $[2,21]$ by comparing, on a relative scale, the measured radiation pattern to several theoretical lines, a further improvement in the accuracy may be obtained by an absolute-scale measurement. For the example in Fig. 5, such improvement may be achieved by positioning a detector at an angle of maximum expected radiation $\theta=12^{\circ}$. Calibrating the detector at a the corresponding wavelength $\lambda \simeq 2 \mathrm{~mm}$, may result in an alternative setup in which the radiation is measured on an absolute scale in order to optimize accelerator parameters to produce the shortest bunch and determine its length without any mechanical sweeping of observation angles as in [21]. Thus, this method may have a better accuracy, be simpler to implement, and will provide a real time, nondestructive, measurement feature.

\section{ACKNOWLEDGMENTS}

This research is supported by the U.S. Department of Energy, Division of High Energy Physics.

[1] S. J. Smith and E. M. Purcell, Phys. Rev. 92, 1069 (1953).

[2] S. E. Korbly, A. S. Kesar, J. R. Sirigiri, and R. J. Temkin, Phys. Rev. Lett. 94, 054803 (2005).

[3] G. Toraldo Di Francia, Nuovo Cimento 16, 61 (1960).
[4] P. M. van den Berg, J. Opt. Soc. Am. 63, 1588 (1973).

[5] O. Haeberlé, P. Rullhusen, J. -M. Salomé, and N. Maene, Phys. Rev. E 49, 3340 (1994).

[6] J. Walsh, K. Woods, and S. Yeager, Nucl. Instrum. Methods Phys. Res., Sect. A 341, 277 (1994).

[7] J. H. Brownell, J. Walsh, and G. Doucas, Phys. Rev. E 57, 1075 (1998).

[8] S. R. Trotz, J. H. Brownell, J. E. Walsh, and G. Doucas, Phys. Rev. E 61, 7057 (2000).

[9] A. S. Kesar, M. Hess, S. E. Korbly, and R. J. Temkin, Phys. Rev. E 71, 016501 (2005).

[10] P. M. van den Berg, J. Opt. Soc. Am. 63, 689 (1973).

[11] A. Gover, P. Dvorkis, and U. Elisha, J. Opt. Soc. Am. B 1, 723 (1984).

[12] Y. Shibata et al., Phys. Rev. E 57, 1061 (1998).

[13] G. Kube et al., Phys. Rev. E 65, 056501 (2002).

[14] K. J. Woods, J.E. Walsh, R. E. Stoner, H. G. Kirk, and R. C. Fernow, Phys. Rev. Lett. 74, 3808 (1995).

[15] J.E. Walsh, Nucl. Instrum. Methods Phys. Res., Sect. A 445, 214 (2000).

[16] A. Doria, G. P. Gallerano, E. Giovenale, G. Messina, G. Doucas, M.F. Kimmitt, H.L. Andrews, and J.H. Brownell, Nucl. Instrum. Methods Phys. Res., Sect. A 483, 263 (2002).

[17] G. Doucas, M. F. Kimmitt, A. Doria, G. P. Gallerano, E. Giovenale, G. Messina, H.L. Andrews, and J.H. Brownell, Phys. Rev. ST Accel. Beams 5, 072802 (2002).

[18] G. Doucas, M.F. Kimmitt, Th. Kormann, G. Korschinek, and C. Wallner, Int. J. Infrared Millimeter Waves 24, 829 (2003).

[19] M. C. Lampel, Nucl. Instrum. Methods Phys. Res., Sect. A 385, 19 (1997).

[20] D. C. Nguyen, Nucl. Instrum. Methods Phys. Res., Sect. A 393, 514 (1997).

[21] S. E. Korbly, A. S. Kesar, R. J. Temkin, and J. H. Brownell (to be published).

[22] J. Haimson, B. Mecklenburg, G. Stowell, and B. Ishii, in Advanced Accelerator Concepts: Tenth Workshop, AIP Conf. Proc. No. 647 (AIP, New York, 2002), p. 810.

[23] J. Haimson, in Advanced Accelerator Concepts: Eleventh Advanced Accelerator Concepts Workshop, AIP Conf. Proc. No. 737 (AIP, New York, 2004), p. 95.

[24] A. S. Kesar, S. Korbly, R. J. Temkin, and M. Hess, in Proceedings of The 2005 Particle Accelerator Conference, Knoxville, Tennessee, 2005 (to be published).

[25] C. A. Balanis, Advanced Engineering Electromagnetics (John Wiley \& Sons, Inc., New York, 1989).

[26] A.F. Peterson, S. L. Ray, and R. Mittra, Computational Methods for Electromagnetics, Electromagnetic Wave Theory (IEEE Press, New York, 1999).

[27] A. W. Mathis and A. F. Peterson, IEEE Trans. Antennas Propag. 44, 567 (1996).

[28] Electromagnetic Theory of Grating, edited by R. Petit (Springer-Verlag, Berlin, 1980). 